\title{
Cooperation without Coordination: Signaling, Types and Tacit Collusion in Laboratory Oligopolies
}

\author{
Douglas Davis \\ Virginia Commonwealth University \\ Richmond VA 23284-4000 \\ (804) 828-7140 \\ dddavis@vcu.edu \\ Oleg Korenok \\ Virginia Commonwealth University \\ Richmond VA 23284-4000 \\ (804) 828-3185 \\ okorenok@vcu.edu \\ Robert Reilly \\ Virginia Commonwealth University \\ Richmond VA 23284-4000 \\ (804) 828-3184 \\ rjreilly@vcu.edu
}

9 December 2008

\begin{abstract}
We study the effects of price signaling activity and underlying propensities to cooperate on tacit collusion in posted offer markets. The primary experiment consists of a baseline sequence that is an extensively repeated variant of a standard posted offer market, and a 'forecast' sequence that adds to the baseline sequence a forecasting game that allows a refined identification of signaling activity. We find that 'forecast' signals differ from standard 'market' signals, and that while both raise market collusiveness, they do so only temporarily. On the other hand, seller propensities to cooperate do affect long-term outcomes. A secondary experiment demonstrates that seller 'type' determines collusiveness.
\end{abstract}

Keywords: Experiments, Tacit Collusion, Price Signaling, Types JEL codes: C9, L11, L13

\footnotetext{
* We thank without implicating Jordi Brandts, Tim Cason, Asen Ivanov and two anonymous referees for helpful comments. Financial assistance from the National Science Foundation (SES 0518829) and the Virginia Commonwealth University Faculty Summer Research Grants Program is gratefully acknowledged. Thanks also to Matthew Nuckols for programming assistance. Experiment instructions, an appendix and the experimental data are available at www.people.vcu.edu/ dddavis
} 


\section{Introduction}

Although not illegal, "tacit” collusion, or coordinated pricing, is a primary concern of antitrust authorities. ${ }^{1}$ In the United States, horizontal merger enforcement activity focuses extensively on preventing the formation of market structures that facilitate tacit collusion. ${ }^{2}$ When assessing the likelihood of coordinated behavior, antitrust authorities attempt to identify factors that affect the capacities of competitors to establish a common price and to develop an arrangement-maintaining enforcement mechanism. ${ }^{3}$

Evaluations of the relative importance of such factors can help inform antitrust policy. Laboratory studies provide a useful complement to more standard empirical and theoretical work on tacit collusion, because the laboratory allows control over costs, demand conditions, information flows and seller communications unavailable in natural contexts. This control allows a direct assessment of the extent of supra-competitive prices and of the factors that facilitate such outcomes. In some laboratory market contexts, such as duopolies and market designs where some or all sellers have unilateral market power, supra-competitive prices arise frequently. ${ }^{4}$ More interesting for the study of tacit collusion are supra-competitive outcomes in market designs where tacit collusion is less expected. Experiments reporting supra-competitive pricing outcomes in 'no power' designs with more than two sellers include Cason and Davis (1995), Cason and Williams (1990), Davis and Korenok (2008), and Durham et al. (2004).

Curiously, the pricing patterns observed in these latter experiments exhibit none of the characteristics that antitrust scholars argue typify tacit collusion. Rather than establishing a common price and coming to some agreement as to how that price is to be

\footnotetext{
1 In contrast, cartelization or 'explicit collusion' is illegal. In the United States, cartelization is illegal per se, and may be prosecuted as a felony, subject to treble damages and prison time.

2 Assessing the likelihood of coordinated pricing post-merger is one of the two primary thrusts of horizontal merger analysis. The other enforcement objective pertains to the likelihood that a proposed consolidation will increase unilateral market power. See, for example, the US Department of Justice and Federal Trade Commission Horizontal Merger Guidelines, 1992 (revised, 1997).

${ }^{3}$ Typically cited 'suspect' factors include product homogeneity, firm symmetry, the frequency of communications, multi-market contacts and the stability of demand.

${ }^{4}$ In a review of experiments that focus on collusion, Haan et al. (2009) concludes “[Experimental] industries with two sellers are able to collude to some extent. Industries with more than two firms are not able to collude at all.” (p. 14). Examples of tacitly collusive outcomes in laboratory market designs with unilateral market power include Davis and Holt (1994) and Davis and Wilson (2000).
} 
maintained, prices more typically vacillate substantially, both within and across markets, with prices ranging from near collusive to competitive levels. ${ }^{5}$

Two explanations for such 'uncoordinated' supra-competitive pricing have some appeal. The first pertains to within-market fluctuations. While sellers may not settle on fixed prices, they may, with the signals and responses sent through their pricing activity, develop a 'language of coordination' that allows the implementation and maintenance of higher prices. The bulk of the pertinent laboratory market studies focus on such signaling behavior. A second possible explanation for supra-competitive pricing pertains to inherent propensities of sellers to cooperate. In experiments studying the voluntary provision of public goods, underlying cooperative propensities have been identified as an important determinant of behavior (e.g., Fischbacher, Gächter and Fehr, 2001, Burlando and Guala, 2005, Kurzban and Houser, 2005 and Gunnthorsdottir, Houser, and McCabe, 2007). Seller 'type’ however, has received almost no consideration as a factor that might explain tacit collusion. ${ }^{6}$

Papers that study signaling activity in pricing games include Holt and Davis (1990), Cason (1995) and Cason and Davis (1995). In each of these studies sellers were given the opportunity to submit non-binding pricing recommendations prior to making binding price offers each period. Results uniformly indicate that such 'cheap talk' signals raise prices, albeit only temporarily. ${ }^{7}$ The limited duration of each of these studies (20 periods or less) represents one possible explanation for the failure of signals to affect overall cooperation levels. More recently Durham et al. (2004) study signaling activity in an experiment where actions are more extensively repeated (80 periods), but where sellers may send signals only via pricing decisions. Durham et al. observe supra-

\footnotetext{
${ }^{5}$ As a theoretical matter, variable prices and quantities may be part of a trigger strategy equilibrium in an environment with demand uncertainty and imperfect information regarding rival's actions (e.g., Green and Porter, 1984). However, we are aware of no model of tacit collusion that has variable prices as part of an equilibrium strategy in a full information, constant cost and constant demand environment, where sellers possess no unilateral market power in the stage game.

${ }^{6}$ Interestingly, the exceptions include some of the very first oligopoly experiments. Fouraker and Segal (1963) classify participants as 'cooperative', 'profit maximizing' and 'rivalistic'. Hoggatt $(1967,1969)$ uses a more continuous classification scheme and examines the effects of 'cooperative' types against robot opponents.

${ }^{7}$ Cason and Davis (1995) do find considerable evidence of persistent tacit collusion in the multi-market context they study, in which sellers were given pre-posting opportunities to submit price signals. However, as in the other papers, signaling opportunities appeared to play, at best, a minor role in raising prices.
} 
competitive prices in many of their markets. ${ }^{8}$ Further, they report some evidence that price signals elicit at least immediate responses. However, while these investigators do not discuss the cumulative effect of signaling activity on cooperation levels, casual inspection of their results again suggests that the correlation between signaling volumes and cooperation levels is quite weak. ${ }^{9}$

The way Durham et al. (2004) measure signaling activity represents one possible reason for the weak link between signaling activity and cooperation levels. Their experiment was not specifically designed to study signaling behavior and to proceed the authors had to construct a relatively imprecise measure of signaling activity, based on the relation between current period price posting decisions and the previous period's prices. As we discuss below, such a definition measures intended signals accurately only when seller expectations regarding rivals’ price decisions are naively adaptive.

This paper reports an experiment designed to assess the effects of price signaling activity and player cooperativeness (type) on tacit collusion in posted offer markets. Our design improves on Durham et al. (2004) for studying signaling activity by including still more extensive repetition and by using a design that allows a more refined measure of signaling activity. We find that nearly all participants send signals and that those signals tend to elicit immediate responses. However, similar to the previous literature we also find little evidence that signaling activity affects the overall collusiveness of markets. On the other hand, seller propensities to cooperate do prominently affect overall collusiveness, and these propensities are relatively stable.

The remainder of this paper is organized as follows. Section 2 overviews the experiment design. Section 3 explains experiment procedures. Section 4 reports experimental results. The paper concludes with a discussion in a short fifth section.

\section{Experiment Design}

We use here a variant of a 'swastika' design initially studied by Smith and Williams (1990) which we examine in an extensively repeated variant of the standard posted-offer institution. Posted offer rules both parallel many features of retail trade, and may be

\footnotetext{
${ }^{8}$ The market structure used by these authors undoubtedly drives some of their results. The experiment design includes fixed costs that assure sellers a loss in the competitive equilibrium.

${ }^{9}$ See in particular the plot of signals and mean transactions prices for each market in figure 5 (Durham et al. 2005, p. 160)
} 
analyzed as a game of Bertrand-Edgeworth competition. Below we explain our implementation of the posted offer trading institution in subsection 2.1. The subsequent subsection 2.2 develops the market design, and subsection 2.3 explains our experimental treatments and conjectures.

\subsection{The 'Near Continuous' Posted Offer Institution}

Under posted-offer rules, the market consists of a series of trading periods. In each period sellers, endowed with unit costs, simultaneously make price decisions. Once all seller decisions are complete, prices are displayed publicly, and an automated buyer program makes all purchases profitable to the buyer at the posted prices. Figure 1 illustrates a screen display for a seller S1 in a computerized implementation of the posted offer market. As seen in the upper left corner of the figure, seven seconds remain in trading period 4. Moving down the left side of the figure, observe that in this period seller S1 has four units, each of which cost $\$ 2.00$. To enter a decision, a seller just types an entry in the 'price' box and presses 'enter'.

In a 'near continuous' variation of this institution, developed recently by Davis and Korenok (2008), the maximum length of decision periods is truncated sharply, to only several seconds. The large number of periods allowed by this 'near continuous' framework very considerably increases sellers' opportunities to both send and respond to price signals.

Numerical and graphical summaries of the previous period's prices and earnings, as shown in Figure 1 help participants to process market results quickly in the nearcontinuous framework. The 'Standing Prices' displayed at center top of Figure 1 indicate that in just completed period 3, S1 posted a price of $\$ 4.20$ while S2 and S3 posted prices of $\$ 4.60$ and $\$ 4.00$, respectively. The bolded bars shown at the bottom center of the figure make it clear that $\mathrm{S} 1$ posted the second highest price in period 3. Further, comparison of the bolded bars to the light gray bars, which illustrate prices for the previous period 2, shows that sellers S1, S2, S3 all reduced their prices in period 3 relative to period 2. In period 3, S1 sold three of the four units he offered and earned $\$ 6.60$ (S1 also earned \$1.00 from the forecasting game, explained below), as shown on the 'Period Earnings' bar chart. The earnings chart also indicates that seller S1's earnings in period 3 fell relative to period 2 . 


\subsection{Market Design}

Figure 2 illustrates supply and demand arrays for the variant of the 'swastika' design used here. Three sellers are each endowed with 4 units with a constant cost of \$2 per unit. Aggregate supply is thus 12 units at prices in excess of $\$ 2$. The (simulated) buyer purchases seven units at any price of $\$ 6$ or less. Our implementation of the 'swastika' design differs from previous implementations in that we impose a minimum price of $\$ 3$ per unit. Given the excess supply of four units, in the competitive equilibrium all sellers post a price of $\$ 3$ and earn strictly positive expected earnings of $\$ 2.33$ per trading period.

The competitive equilibrium is the unique Nash equilibrium for the market evaluated as a stage game. To see that this outcome is an equilibrium, observe that earnings will fall to zero for any seller who unilaterally raises price above a common price of $\$ 3$. For uniqueness, observe that at common price above $\$ 3$ a seller could increase earnings by posting a price $1 \mathbb{\$}$ below the common price. For any vector of heterogeneous prices above $\$ 3$, the highest pricing seller will sell nothing.

This design is useful for studying price signaling, for three reasons. First, previous research indicates that this design frequently stimulates tacit collusion (Cason and Williams, 1990, Davis and Korenok, 2008). Second, the simple demand and cost conditions help participants understand underlying market structure, thus reducing the number of initial periods participants need to appreciate the incentives that the design induces. Third, this design, in conjunction with a forecasting treatment described below, helps isolate signaling activity.

\subsection{Treatments and Conjectures}

Our primary experiment consists of two treatments: a 'baseline' treatment, and a 'forecasting' treatment.

\subsubsection{Baseline Treatment}

In the baseline treatment, participants make price decisions in an extensively repeated market, using the supply and demand arrays shown in Figure 2. Each period lasts a maximum of 12 seconds. Decisions in the baseline treatment allow us to verify that tacit collusion persists in this variant of the swastika design. In particular, we are concerned that the guarantee of positive earnings induced by our inclusion of a minimum admissible 
price does not undermine the tacitly collusive behavior observed in other markets. This is our first conjecture.

Conjecture 1(a): Tacit collusion in our variant of the 'swastika' design is resilient to the inclusion of a minimum admissible price that guarantees a positive profit.

Results of the baseline treatment are further useful in that they allow an analysis of the effects of other treatments, as discussed below.

\subsubsection{Forecasting Treatment}

To study signaling behavior, Durham et al. (2004) define a price signal as "any price submitted by any firm that is greater than or equal to the lowest posted price that failed to attract buyers in the previous period” (p. 155). This 'market-based' definition, while quite natural, suffers the potential deficiency that it accurately identifies signals only when sellers have naively adaptive expectations, i.e., expectations based only on the immediately preceding period outcomes. If sellers form expectations differently, this market-based definition may both include some price postings that were not intended to be signals, and may exclude other postings that were. For example, if prices are trending upward, a market-based measure may errantly include as signals those prices posted by sellers who expect rivals' to continue raising their prices. Similarly, if prices are trending downward, a market-based definition would miss any signals sent by a seller who expects rivals to post lower prices, and attempts to interrupt or slow the downward trend by submitting price postings that either maintain current price levels, or decrease the price level by less than rivals.

In an effort to relax the naively adaptive expectations assumption and thus more definitively isolate price signaling behavior, we introduce a 'forecasting' treatment where we elicit sellers' expectations. In this treatment, sellers predict the maximum price their rivals will post in the subsequent period. If a seller’s forecast is within $5 \mathbb{4}$ of the subsequently observed maximum price posted by rivals, the seller earns a 'high' forecast

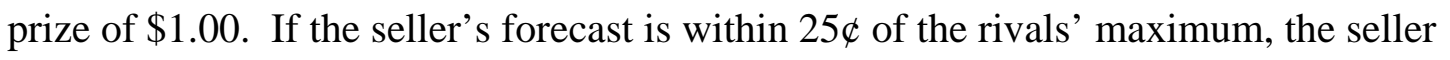
earns a 'low' forecast prize of 50థ. Otherwise the forecast prize is zero.

A review of the screen display in Figure 1 illustrates the presentation of the forecasting game to sellers. After posting a price, the cursor moves to the 'forecast' box. The seller then enters a price forecast and presses 'enter'. Forecast earnings are 
illustrated graphically as supplements to the period earnings bar. For example, in Figure 1, S1's forecast for period 3 was within 54 of the rivals’ maximum $\$ 4.30$ so seller S1's earnings for period 3 are supplemented by $\$ 1.00$, as indicated by the supplemental shaded box in the seller's earnings chart. ${ }^{10}$

Although we did not expect the addition of the forecasting feature to affect outcomes, we recognize that such factors may have an impact. ${ }^{11}$ In particular, Croson (2000) observes that asking players to submit beliefs about the likely contributions levels of others (analogous to posting forecasts of others' prices here) tends to reduce group contributions in a voluntary contributions game. ${ }^{12}$ As a second conjecture we explore whether or not our forecasting procedure tends to reduce 'collusive efficiency,' or the extent of tacit collusion in a market.

Conjecture 1(b). Relative to the initial sequence, eliciting forecasts does not affect collusive efficiency.

The forecasting game, in conjunction with a design where the highest pricing seller sells zero units, allows a clean identification of an intended signal: any price above a seller's forecast maximum price will result in sales of zero, and would be irrational unless the seller intends to send a signal. ${ }^{13}$ To the extent that the forecasts allow a more precise

${ }^{10}$ Our introduction of a forecasting game emulates the expectations elicitation techniques used in some early asset market experiments (e.g., Williams, 1987 and Smith, et al. 1988). Concerns about biasing pricing behavior with the forecasting game are somewhat diminished relative to this earlier literature because sellers here are unable to use their market decisions to influence their chances of winning the forecasting game.

${ }^{11}$ One additional minor procedural difference between the baseline and forecasting markets is that we obligated sellers to enter a new price and forecast each period, even if they wanted to repeat the same posting. (Absent this requirement, we might lose information regarding forecasts in those periods where sellers decided they didn't want to post a new price). This difference is minor because participants tended to enter new price (and forecast) entries each period even when they repeated their previous period decisions. Periods ended either with the expiration of the maximum time limit, or when all participants posted prices. In both sequences, due either to impatience or an effort to increase hourly earnings, periods virtually always ended prior to the time deadline as a result of all sellers posting a price. Identification of sellers who changed price frequently in each treatment provides further evidence that the reposting requirement in the forecasting treatment did not stimulate added price volatility. In the baseline sequences, the average seller posted new prices in $76.3 \%$ of periods where prices were not initially competitive. In the forecasting sequences the average seller changed prices an almost identical $78.5 \%$ of periods.

${ }^{12}$ In a related experiment Wilcox and Feltovich (2002) were unable to replicate the Croson result. However, as Wilcox and Feltovich observe, their 'elicitation' treatment differed from Croson in that they require participants only to guess the number of players who would submit positive contributions each period, rather than the expected contributions levels.

${ }^{13}$ Conceivably, a risk-taking seller might post a price that exceeds his forecast (the expected maximum) placing a high weight on the potential for the rival maximum to fall above his expectations. For such an action price to be optimal, however, the seller must be extremely risk-preferring. As shown in table A1 of 
identification of price signaling activity, we can examine the effects of our refined definition of signaling.

Conjecture 2: Forecast-based and market-based measures of signaling activity differ significantly.

We also explore the relationship between signaling and supra-competitive prices.

Conjecture 3: Price signaling activity affects tacit collusion.

Two separate dimensions of conjecture 3 merit discussion. First is the question of whether or not rivals recognize signals and respond to them with higher prices. If rivals fail to understand a signaler's intended communications, an enhanced capacity to identify when signals are sent may translate into relatively little in the way of price responses. The second dimension regards the link between signaling activity and collusive efficiency levels. Even if rivals tend to respond immediately to signals, it is not obvious that signals will have a lasting impact on overall collusiveness. Sellers, for example, might respond to signals but then quickly undercut each other, resulting in a fairly competitive price history. We will assess both dimensions of conjecture 3 .

\subsubsection{Collusive Efficiency and Propensities to Cooperate}

Market outcomes may also be importantly affected by the inherent propensities of participants to cooperate or compete. We assess this propensity by examining whether sellers aggressively undercut standing prices. In a market populated by sellers who do not aggressively undercut each other, even one signal might be sufficient to achieve a high degree of collusion. On the other hand, if sellers aggressively undermine each others' attempts to cooperate, even frequent signals may fail to sustain a high degree of collusiveness.

Here we classify participants endogenously on the basis of their decisions in the market, as has been done frequently in the VCM literature (e.g., Kurzban and Houser, 2005). A variety of other methods, including strategy elicitation (Fischbacher et al. 2001), elicitation of cooperative preferences in a related game (Offerman et al., 1996), and the use of questionnaires (Burlando and Guala 2004) have also been used in the

an online appendix, the costs of such 'hedging' activity are at least half of the expected earnings from posting the profit maximizing price under a variety of distributional assumptions regarding a seller's beliefs about the expected actions of rivals. 
VCM literature. However, given our uncertainty as to the characteristics that drive cooperative behavior in a market game, a classification scheme based on previous decisions in a similar environment seems a most reasonable starting point. High collusive efficiency levels in markets populated by sellers who are on average 'cooperative' would be an indication of a link between propensities to cooperate and collusiveness. This is a fourth conjecture.

Conjecture 4: Markets comprised of sellers who are on average more cooperative have higher collusive efficiency levels than markets comprised of sellers who are on average less cooperative.

Given the endogenous nature of our cooperativeness measure, a natural concern is whether it assesses cooperativeness or simply reflects market performance. If our measure of cooperativeness is a lagging measure of market performance, cooperativeness is an effect of collusive efficiency, not a cause of it. To establish more clearly a causal link, we conducted a follow-up experiment that duplicated the initial experiment except that participant type was homogenous within cohorts. If type determines performance and is relatively stable across sessions, we should observe relatively higher collusive efficiencies in markets composed of cooperative types than in the markets composed of more competitive types. If types, however, simply reflect market performance, then a cooperative index in initial sessions will not be able to explain cooperation levels in new sessions. This is a fifth conjecture

Conjecture 5: Individual 'types' or propensities to cooperate determine collusive efficiency levels.

\section{Experiment Procedures}

To evaluate the above conjectures we conduct the following experiment. Nine (and in one instance twelve) player cohorts are invited into the laboratory to participate in three (four) triopolies. At the outset of each session a monitor randomly seats participants at visually isolated computers. The monitor then reads aloud instructions, as the participants follow along on printed copies of their own. Each session consists of two 120 period sequences, a baseline sequence and a forecasting sequence. Prior to the first sequence, instructions explain price-posting procedures, as well as the minimum admissible price of \$3.00. Participants are also given as common knowledge full 
information regarding aggregate supply and demand conditions shown in Figure 2. To ensure that participants understand these underlying conditions the monitor elicits responses to a series of possible price postings. After giving participants an opportunity to ask questions, the first sequence begins, and consists of 120 periods each with a maximum length of 12 seconds. After period 120, the baseline sequence is terminated without prior announcement, and participants record their earnings.

Following the first sequence, a monitor remixes participants into entirely new groups, and reads instructions for a second sequence. ${ }^{14}$ Conditions for the second sequence match those in the first, except that the maximum period length is increased to 18 seconds and the forecasting game is added. After giving participants time to ask questions, the second sequence began. After 120 periods, this sequence ended, again, without prior announcement. Participants were paid privately the sum of their earnings for the two sequences, converted at U.S. currency at a rate of $\$ 100$ lab $=\$ 1$ U.S. plus a $\$ 6$ appearance fee, and were dismissed one at a time.

Participants were volunteers recruited primarily from upper level undergraduate business and economics classes at Virginia Commonwealth University. All participants were 'experienced' in the sense that they had previously participated in a near continuous posted offer session, but for a different study, with different supply and demand conditions. No one participated in more than one session.

Following the initial sessions, we ranked participants by their cooperativeness 'type' and invited groups of nine homogenously cooperative and nine homogenously competitive participants to participate in a subsequent session. Other than the homogeneity of participant types, procedures for the 'experienced' pair of sessions matched exactly the procedures in the initial sessions. The fact that participants were of like types was not revealed to participants.

Earnings for the initial sessions, which lasted between 80 and 100 minutes, ranged from $\$ 21$ to $\$ 46$ and averaged about $\$ 31$. In the two experienced sessions, earnings ranged from \$21 to \$57 and averaged \$37.

\footnotetext{
${ }^{14}$ In the nine person cohorts, for example, markets in the initial sequences are ordered $(1,2,3),(4,5,6)$ and $(7,8,9)$. In the second sequence markets shift to $(1,4,7),(2,5,8)$ and $(3,6,9)$. Thus, for the second sequence, each participant is in a market with participants he or she has not been paired with previously.
} 


\section{Results}

We present data in terms of a collusive efficiency index, which in period $t$ of market $j$ is defined as $\psi_{j t}=\frac{\pi_{j t}-\pi_{N E}}{\pi_{J P M}-\pi_{N E}}$ where $\pi_{j t}$ denotes realized aggregate period profits, $\pi_{N E}$ denotes static Nash (competitive) profits and $\pi_{J P M}$ denotes earnings at the joint maximizing level. Collusive efficiency values range between zero at the competitive level and one in the joint profit maximizing outcome. In our design $\psi_{j t}$ closely parallels mean transaction price paths. ${ }^{15}$

Figure 3 provides an overview of results for the baseline and forecasting sequences, respectively. Inspection of the panels in Figure 3 provides information pertinent to conjectures $1(\mathrm{a})$ and $1(\mathrm{~b})$. The clearly positive $\bar{\psi}_{t}$ values for the baseline sequences indicate that markets do not collapse on competitive outcomes. Nevertheless, we recognize that classifying market outcomes as either 'collusive' or 'competitive' is to some extent arbitrary. No well-defined standard has emerged from the behavioral oligopoly literature that identifies the level of deviations from static Nash predictions necessary for an outcome to be 'collusive.' Sellers in laboratory markets rarely settle on zero variance outcomes, and the current environment is no exception. In both our more 'competitive' and more ‘cooperative' market sequences, high outcome variability was a persistent feature. $^{16}$

To distinguish this inevitable noise from sellers’ success in raising prices, we adopt the convention of defining a segment of periods as 'collusive' if $\bar{\psi}>.10$, meaning that sellers on average extract at least $10 \%$ of the supra-competitive profits available from joint maximization. As indicated by the entries in column (4) of Table 1, using this measure the null hypothesis that baseline markets are competitive, can be rejected with

\footnotetext{
${ }^{15}$ In our market design collusive efficiency is essentially a linear transformation of mean transaction prices (e.g., $\left(\bar{p}_{j t}-3\right) /(6-3)$, where 6 and 3 are joint maximizing and competitive prices, respectively. The only deviation occurs when more than one seller posts a price above $\$ 6$ in a period. We evaluate results in terms of collusive efficiency rather than transaction prices in order focus on sellers' relative success as tacit conspirators. This 'collusive efficiency' index has been used to calculate the efficiency of tacit collusion and monopoly power exercise in a variety of contexts, under titles such as 'cooperativeness' (Potters and Suetens, 2008) and monopoly efficiency index (e.g., Isaac, Ramey and Williams, 1984).

${ }^{16}$ For example, only one of the 32 combined baseline and forecast sequences stabilized on a single-price outcome (this was a competitive baseline sequence). Mean contract price plots for individual baseline and forecast markets appear as Figures A-1 and A-2 in an online appendix.
} 
high significance for periods 1-60, periods 61-120, and overall, using the Wilcoxon test. ${ }^{17}$ At the same time, sellers in the baseline sequence were rarely particularly efficient conspirators. As seen in column (2) of Table 1, average collusive efficiency levels reached only 0.32 in the last 60 periods of the baseline sequences. Finally, as suggested by the breadth of the inter-quartile ranges for mean collusive efficiency rates, shown in Figure 3, outcomes varied widely across sequences. For the baseline sequences, $\bar{\psi}_{j}<0.1$ in two of the 16 baseline sequences, and $\bar{\psi}_{j}>0.5$ in another three sequences. Combined, these results represent a first finding.

Finding 1(a): Mean collusive efficiency levels above those consistent with a competitive outcome are frequently observed in our version of the swastika design where sellers earn strictly positive earnings in the competitive equilibrium.

Mean and inter-quartile ranges for collusive efficiency in the forecasting sequences, shown in the lower panel of Figure 3, parallel results for the baseline markets. Sellers again tended to achieve supra-competitive outcomes. As summarized in column (5) of Table 1, using the Wilcoxon test the null hypothesis that collusive efficiency is $10 \%$ or less can be rejected with high significance for the segments consisting of periods 1-60, periods 61-120, and overall. However, as with the baseline sequences, the width of the inter-quartile ranges in the forecast sequences again suggests that outcomes were quite variable across markets. For example, in the forecasting treatment $\bar{\psi}_{j}<0.10$ in two instances and $\bar{\psi}_{j}>0.50$ in six instances.

Comparing the upper and lower panels of Figure 3 suggests that sellers exhibited somewhat higher collusive efficiencies in the forecasting sequences, particularly in the initial 60 periods. However, as indicated in column (6) of Table 1, the data are too dispersed to conclude that these differences are significant. Further, comparing mean entries in columns (2) and (3), observe that the overall lower collusive efficiencies of the baseline sequences are driven largely by outcomes in the first 60 periods. Since the baseline sequences uniformly preceded the forecasting sequences, the comparatively lower initial collusive efficiencies in the baseline sequences may quite possibly be attributable to learning effects. In any case, the forecasting treatment clearly does

\footnotetext{
${ }^{17}$ In the discussion of results we term results as 'highly significant' if $p<.01$, 'significant' if $p<.05$ and 'weakly significant' if $p<.10$.
} 
nothing to dampen prices in this design, as results by Croson (2000) would suggest. This is a second result.

Finding $\mathbf{1 b}$ : The addition of a forecasting treatment does not significantly affect collusive efficiency.

These initial results allow us to focus more specifically on the larger issues: the effects of signaling and propensities to cooperate on collusive efficiency. We consider first signaling behavior.

\subsection{Signaling and Collusive Efficiency}

In this subsection we first compare our results with previously generated results by examining market-based signals and the effects of these signals on collusive efficiency. Then we consider why the accuracy of this measure might be questionable, and evaluate an alternative forecast-based signal.

\subsubsection{Market-Based Signals}

Analogous to Durham et al. (2004) we define a 'market-based' signal (' $s_{m}$ ') as a price that either exceeds either the maximum price posted in the previous period, or the $\$ 6.00$ limit price. ${ }^{18}$ A first pertinent question regarding such signals is whether or not they elicited responses from sellers. Columns 1 and 2 of Table 2 present results of a regression of lagged transaction prices and signals, sent under definition $s_{m}$ for the baseline and forecasting sequences, respectively. Specifically, we estimate

$$
p_{j t}=c+\beta_{p j} p_{j t-1}+\beta_{s j} I_{s m, j t-1}+\varepsilon_{j t},
$$

where $I_{s m, j t}$ is an indicator variable of the market-based signal sent in market $j$ during period $t$. To control for possible interdependencies within markets, we cluster by markets and estimate robust standard errors. As the large and highly significant coefficients on the $I_{s m}$ variable suggest, actions classified as signals sent under $s_{m}$ tend to elicit next period responses from sellers, both in the baseline sequences (summarized in column 1) and in the forecasting sequences (shown in column 2). These results closely parallel results observed previously by Durham et al. (2004).

\footnotetext{
${ }^{18}$ In our design, the maximum price posted in a period is the lowest price that failed to yield a transaction (unless the maximum price was shared by two sellers). Price postings above \$6 can uniformly be interpreted as signals since sellers know that the buyer will purchase no units at such prices.
} 
A broader question regards the relationship of signaling activity to collusion levels. To assess the relationship between signaling activity and overall collusive efficiency, we regress the number of $s_{m}$ signals on mean collusive efficiency values for each market $\bar{\psi}_{j}$ for the initial and forecasting treatments combined. As a check for possible treatment effects we introduce both intercept (' $f c s t$ ') and slope (' $s_{m} \times f c s t$ ') terms for the forecasting sequences, as summarized in equation (2).

$$
\begin{aligned}
& \hat{\bar{\psi}}_{j}=0.27^{*}+0.25 f c s t+0.11 s_{m}-0.66\left(s_{m} \times f c s t\right)+\varepsilon_{j} \quad \bar{R}^{2}=-0.01 \\
& \text { (0.14) (0.19) (0.53) } \\
& n=32
\end{aligned}
$$

As is clear from equation (2), signal volumes collected under $s_{m}$ organize essentially none of the variation in collusive efficiencies observed across sessions. Other than the intercept, none of the individual parameters are significant, and for the regression as a whole, $\bar{R}^{2}=-0.01$. $^{19}$

\subsubsection{Are Seller Expectations Naively Adaptive?}

A 'market-based' signal definition implicitly assumes that seller expectations about rivals' actions are naively adaptive. The forecasting treatment allows insight into the extent to which this assumption is correct: a seller has naively adaptive expectations if the seller's forecast of his or her rivals' maximum price equals the maximum price that rivals posted in the previous period. However, a comparison of forecasts and previous period postings in the forecast sequences suggest that this is not the case. ${ }^{20}$ Overall, sellers submitted forecasts consistent with naively adaptive expectations only $23 \%$ of the time. Further, many of these consistent choices occurred in markets where prices had collapsed on the \$3 competitive outcome. Confining attention to those periods where the maximum rivals’ price exceeded $\$ 3$, sellers made choices consistent with naively adaptive expectations only $11 \%$ of the time. That seller expectations are generally not naively adaptive provides some basis for skepticism regarding 'market-based' signaling measures of signaling activity.

\footnotetext{
${ }^{19}$ One obvious possible reason that market-based signal volumes may fail to explain collusive efficiency is that some market-based signals, may be too subtle for rivals to observe and respond to. One possible alternative market-based definition of a signal would be to confine attention to 'large' signals. As reported in Table A2 of an online appendix, confining attention to 'large' market-based signals (e.g., signals in excess of 50\$) improves the relation between signal volumes and collusive efficiency modestly at best.

${ }^{20}$ A histogram plotting the frequency of forecasts consistent with naively adaptive expectations appears as Figure A4 of an online appendix.
} 


\subsubsection{Forecast-Based Signals}

As an alternative to 'market-based' signals, we may in the forecasting sequences measure signaling activity in terms of instances where sellers' prices exceed their forecasts of the maximum price posted by rivals in the upcoming period. Formally, define a 'forecast signal' (' $s$ ') as a price that is either in excess of a seller's forecast of rivals' maximum posted price for a period or above $\$ 6.00$.

The histogram in Figure 4 plots the incidence of forecast and market signals in the 16 forecasting sequences. As seen in the histogram, signaling activity under the two measures differs substantially. While sellers on average sent less than 10 signals per sequence under $s_{m}$, they averaged nearly 21 per sequence under $s_{f}$. Further, signaling activity under $s_{f}$ is far more dispersed. Under $s_{f} 13$ of 48 participants sent 30 or more signals, while under $s_{m}$ no one sent more than 29 signals.

The higher incidence of signals under $s_{f}$ suggests that sellers' interests in cooperation may considerably exceed the levels indicated by $s_{m}$. Both $s_{f}$ and $s_{m}$ capture efforts to encourage other sellers to raise price from a low level. However, only $s_{f}$ can capture signals intended to retard the erosion of market prices from a high level. Further, unlike signals sent under $s_{f}$, many price postings interpreted as signals under $s_{m}$ were 'unintended' in the sense that a seller expected rivals' prices to be higher than the previous period's maximum price. As seen in row (2) of Table 3, 28.3\% of the signals sent under $s_{m}$ (128 of 453) were 'unintended' in the sense that a seller posted a price above the market maximum for the previous period, but below their forecast of their rivals' maximum price. On the other hand, while the $s_{f}$ signal definition reveals a high frequency of signaling, we also observe that the intended recipients miss many such signals. If a signaler underestimates the expected maximum price of his rivals, then his signal may fall below the actual maximum in the upcoming period, thus masking his signal. As summarized in row (3) of Table 3, 27.6\% of signals sent under $s_{f}$ (275 of 998) were 'masked' in the sense that the signal price is below the maximum price charged by rivals in the period of the signal. Nearly $15 \%$ of signals sent under $s_{m}$ (62 of 453) were similarly 'masked'. Given the many inconsistencies in $s_{m}$ and $s_{f}$ signals, it is not surprising that $67.4 \%$ of signals sent under $s_{f}(674$ of 998$)$ were missed by measure $s_{m}$, 
and that the average simple correlation between $s_{m}$ and $s_{f}$ is only 0.41 , as shown in row (5). These observations form a second finding.

Finding 2: A signaling definition based on deviations from forecasts (' $\mathrm{s} f$ ') measures activity that differs substantially from a measure based on deviations from previous market prices (' $\mathrm{s}_{m}$ '). Measure $\mathrm{s}_{f}$ suggests that cooperative intentions may be considerably more intense that those measured under $s_{m}$.

Consider now the relationship between signaling activity measured under $s_{f}$ and prices or collusive efficiency. A first question regards whether the signals sent under $s_{f}$ elicit immediate responses, as was observed in equation (1) for $s_{m}$. Regression results reported in column (3) of Table 2 indicate an affirmative answer. The coefficient on the $I_{s f}$ dummy (0.18) is again highly significant. We note that this coefficient is quantitatively smaller than its $I_{s m}$ counterpart for the forecast sequences (0.32). This difference, however, is unsurprising in light of the many and often smaller signals sent under $s_{f}$.

Nevertheless, we unfortunately still find little relationship between collusive efficiency levels and signaling volumes under $s_{f}$. Regressing collusive efficiencies on the incidence of signal volumes under $s_{f}$, for the forecasting sequences yields.

$$
\begin{aligned}
& \hat{\bar{\psi}}_{j}=0.30^{*}+0.17 s_{f j}+\varepsilon_{j} \quad \bar{R}^{2}=-0.06 \\
& \text { (0.21) (0.38) } \quad n=16
\end{aligned}
$$

As was the case for equation (2), only the intercept in equation (3) is even weakly significant. The $\bar{R}^{2}=-.06$, suggests that the regression explains essentially none of the variation in sales. ${ }^{21}$

One potential problem with $s_{f}$ is that although it may reflect an increased intensity of seller interest in behaving cooperatively, it may err in that in a great many instances sellers fail to communicate their intentions. We investigated a variety of possible ways to restrict attention to those forecast signals that were communicated to other sellers, including those forecast signals that were 'large’ (exceeded their forecast by at least 50 cents), those that were 'persistent' (were repeated at least three times) and those that were 'communicated' (either exceeded the forecasts of other sellers or were followed by an increase in rivals' forecasts). As reported in table A3 of an online appendix, none of

\footnotetext{
${ }^{21}$ Notice that regression equation (3) involves only the forecasting sequences. For that reason, it does not include intercept and slope dummies that distinguish baseline and forecast sequences, as in (2).
} 
these modifications to $s_{f}$ improve the capacity of signal volumes to organize collusive efficiency levels. These observations form our third finding.

Finding 3: Price signals, measured either as market-based signals or as forecast-based signals tend to elicit increases in transaction price responses in the immediately following periods. However, signal volumes under either measure are essentially uncorrelated with overall collusive efficiency levels.

\subsection{Types and Collusive Efficiency}

In this subsection we define a measure of 'type', show that it is highly correlated with market's collusive efficiency and, finally, demonstrate that 'type' determines collusive efficiency.

As was the case in our discussion of signaling activity, cooperation may potentially be measured in a number of ways. Among other possibilities, 'cooperative' sellers may be those who tend not to undercut the maximum price in the immediately preceding period, those who tend to shade under the maximum price in the preceding period by only a small margin, or those who tend not to undercut the second highest price in the preceding period. In Table A4 of an online appendix we consider each of these possibilities and find that a cooperativeness measure based on a seller's tendency to undercut the previous period maximum only slightly at most, best organizes observed collusive efficiency levels. ${ }^{22}$

More precisely, we define a 'price leader minus five', or $p_{l-5}$ index as the percentage of periods that a seller posted a price above $\$ 3$, and was either above $\$ 6$, or no more than $5 \mathbb{C}$ below the previous period maximum price. Figure 5 plots mean collusive efficiencies against the average $p_{l-5}$ values per market, $\bar{p}_{l-5 j}$. The scattergram illustrates the impressive organizing power of the $p_{l-5}$ measure. Regressing the observed collusive efficiency levels on $\bar{p}_{l-5 j}$ for each market $j$ yields the estimate summarized as equation

$$
\begin{array}{ccc}
\hat{\bar{\psi}}_{j}=-0.14+0.11 f C S t+2.09^{* * *} \bar{p}_{l-5 j}-0.26\left(p_{l-5 j} \times f C S t\right)+\varepsilon_{j} & \bar{R}^{2}=0.63 \\
(0.11)(0.13) & (0.50) & (0.59)
\end{array}
$$

\footnotetext{
${ }^{22}$ The increase in explanatory power associated with using the $p_{l-5}$ measure over the alternatives mentioned in the text is marked. Regressions of collusive efficiency on these alternative measures of 'type', similar to the regression reported in equation (5) yield no individual parameter estimates that approach significance, and in each case $\bar{R}^{2} \leq 0.18$.
} 
The large and highly significant coefficient on $\bar{p}_{l-5 j}$ in equation (4) reflects the high correlation between mean $p_{l-5}$ values for each market and collusive efficiencies. Observe further that the forecast treatment does not significantly affect either the intercept (fcst) or the slope (fcstxslope) of this relationship. As indicated by $\bar{R}^{2}$ in (4) the regression explains $63 \%$ of the variation in mean collusive efficiencies.

Further, the simple correlation of individual $p_{l-5, i}$ measures across sequences, $\rho$ $=.55(p<.001)$, suggests that the $p_{l-5}$ index is fairly stable across sequences within sessions. Given that sellers are remixed into completely different groups each sequence, this correlation may understate the stability of seller propensities to act cooperatively. A seller may be quite cooperative by nature, for example, but may give up on cooperative efforts in a sequence where her rivals are extremely competitive.

The impressive capacity of mean $p_{l-5}$ values to organize observed cooperation levels and their stability across sequences after random regrouping forms our fourth finding.

Finding 4. Collusive efficiencies are higher in markets with sellers who are on average more cooperative than in markets with sellers who are on average less cooperative. Participant types are reasonably stable across sequences within sessions.

\subsubsection{Type, Collusive Efficiency and Causality}

Our follow up experiment consisted of two additional nine participant sessions, one in a 'cooperative' cohort and the other a 'competitive' cohort. In an effort to demonstrate that 'type' determines collusive efficiency, we classify individuals on the basis of their average $p_{l-5}$ measures in the first two sequences. Using this measure, we were able to get reasonable type separation. Overall, $\bar{p}_{l-5, i}$ values in the first session ranged from 0.03 to 0.55 and averaged 0.22. For the nine participants in our 'competitive' cohort, the index averaged 0.13 , with a maximum of 0.18 . In our 'cooperative' cohort, the index averaged 0.39 with a minimum of 0.25 . Except for the homogeneity of types within groups (which was not known to participants) procedures for these second sessions duplicated exactly those used in the initial experiment.

Figure 6 and Table 4 summarize results of these out-of-sample markets. Figure 6 plots $\bar{p}_{l-5, i}$ values in the second session against comparable values in the initial session. 
Type appears to be quite stable across sessions. Initially competitive and cooperative types form separate clusters in the second session roughly along the $45^{\circ}$ line. The correlation between initial session and second session $\bar{p}_{l-5, i}$ values is a highly significant $\rho=0.69(p<0.001)$.

Differences in propensities to cooperate powerfully impact collusive efficiency levels. Table 4 shows mean collusive efficiency levels in the six competitive and six cooperative second session markets relative to the 32 markets in the initial session. ${ }^{23}$ The mean collusive efficiency level in the six markets with 'cooperative' types (76.4) is more than double the mean rate in the 32 initial sessions (34.2) and almost four times the mean cooperation rate in the six markets with competitive types. These results combine to form a fifth finding.

Finding 5: Sellers' propensities to cooperate determine collusive efficiency. Out-ofsample, markets composed of homogeneously cooperative types exhibit higher collusive efficiency levels than markets comprised of homogenously competitive types.

\section{Discussion}

This paper investigates two dimensions of behavior that may explain tacit collusion in the absence of any obvious coordinating mechanism: price signaling activity and a propensity for participants to behave cooperatively or competitively. With regard to signaling, even with extensive repetition and an enhanced capacity to identify signals, we are unable to find any obvious simple link between signaling activity and collusiveness. On the other hand, we find that 'type' is relatively stable across markets, and that type variations very prominently affect collusiveness.

Our findings are important for two reasons. First, 'type' represents an identifiable determinant of tacit collusion within the laboratory. Failure to control for type will at least significantly increase the variability of market outcomes, and will bias results if subjects are drawn from populations that are not random with respect to type.

Second, and more generally, although sellers in natural contexts may certainly develop a 'language of coordination’ via their pricing activity, our results suggest that at least in some situations an underlying propensity for sellers to cooperate may sustain

\footnotetext{
${ }^{23}$ Notice in Table 4 that we treat the three baseline and three forecasting sequences in each session as independent observations. Our re-matching of individuals across sequences into groups of players with which no seller had previous contact makes this treatment reasonable.
} 
collusion absent the at least obvious development of such a language. For that reason, attention to characteristics that may reveal a propensity to cooperate, in the sense that sellers do not aggressively undercut rivals, may be merited. In this respect our results are consistent with the call by Baker (2002) for increased attention to 'maverick' firms in horizontal merger analysis. For example, in our markets, although cooperative types were sometimes able to maintain quite high levels of collusive efficiency in homogenousgroup markets, cooperators benefited considerably from the elimination of low pricing 'mavericks.' For the nine 'cooperative' sellers who we invited back to participate in a second session, collusive efficiencies for the homogenous markets in which they participated were $37 \%$ higher, and earnings $87 \%$ higher than those in the initial mixed markets.

In closing we mention two areas for future investigation regarding the relationship between type and market performance. First, an investigation of the factors underlying cooperativeness is merited. Cooperative sellers may be driven by some sense of altruism, as is frequently the focus of discussion in the VCM literature. However, successful cooperation in a market context is personally profitable, and so other factors such as strategic sophistication, low discount rates, and preferences for risk are also potentially influential explainers. Identifying the mix of factors consistent with cooperativeness might help identify characteristics of sellers prone to tacit collusion in natural contexts. Second, it would be useful to examine the importance of types in environments less conducive to tacit collusion. Our market, with a small number of symmetric sellers who interact extensively, contains a number of 'facilitating' factors. Exploration of the importance of type in environments that are less conducive to tacit collusion would be of some policy interest as it would allow some assessment of the importance of type relative to the more standard structural indicators of tacit collusion.

\section{References}

Baker, Jon (2002) "Mavericks, Mergers and Exclusion: Proving Coordinated Competitive Effects Under the Antitrust Laws,” New York University Law Review 77:135-203.

Burlando, Roberto, M. and Francesco Guala (2005) “Heterogeneous Agents in Public Goods Experiments” Experimental Economics, 8, 35-54 
Cason, Timothy N. (1995) "Cheap talk Price signaling in Laboratory Markets,” Information Economics and Policy, 7, 183-204

Cason, Timothy N. and Douglas D. Davis (1995) "Price Communications in a MultiMarket context: An Experimental Investigation," Review of Industrial Organization, 10, 769-787.

Cason Timothy and Arlington W. Williams (1990) "Competitive Equilibrium Convergence in a Posted-Offer Markets with Extreme Earnings Inequities" Journal of Economic Behavior and Organization, 14, 331-352

Croson, Rachael. (2000) “Thinking Like a Game Theorist: Factors Affecting the Frequency of Equilibrium Play, Journal of Economic Behavior and Organization 41:299-314.

Davis, Douglas D. and Charles A. Holt (1994) "Market Power and Mergers in Laboratory Markets with Posted Prices,” RAND Journal of Economics, 25, 467-487.

Davis, Douglas D. and Oleg Korenok (2008) "Posted-Offer Markets in Near Continuous Time: An Experimental Investigation” Economic Inquiry. Forthcoming

Davis, Douglas D. and Bart J. Wilson (2000) “Cost Savings and Market Power Exercise”, Economic Theory 16, 545-565

Durham, Yvonne, Kevin McCabe, Mark A. Olson, Stephen Rassenti and Vernon Smith (2004) "Oligopoly Competition in Fixed Cost Environments" International Journal of Industrial Organization 22, 147-162

Fischbacher, Urs, Simon. Gächter and Ernst Fehr (2001) “Are People Conditionally Cooperative? Evidence from a Public Goods Experiment” Economics Letters, 71, 397-404.

Fouraker, Lawrence E., and Sidney Siegel (1963) Bargaining Behavior, New York: McGraw Hill.

Green, Edward J., and Robert H. Porter (1984) "Noncooperative collusion under Imperfect Price Informations,” Econometrica, 52, 87-100.

Gunnthorsdottir, Anna, Danial Houser, and Kevin McCabe (2007) "Disposition, History and Contributions in Public Goods Experiments" Journal of Economic Behavior and Organization, 62, 304-315.

Haan, Marco A., Lambert Schoonbeek and Barbara M. Winkel (2009) "Experimental Results on Collusion. The Role of Information and Communication.” In J. Hinloopen and H. T. Normann (eds.), Experiments and Competition Policies, Cambridge University Press (forthcoming) 
Hoggatt, Austin C. (1969) "Response of Paid Students to Differential Behavior of Robots in Bifurcated Duopoly Games,” Review of Economic Studies, 36, 417-432

Hoggatt, Austin C. (1967) "Measuring Cooperativeness of Behavior in Quantity Variation Duopoly Games,” Behavioral Science 12, 109-121.

Holt, Charles A. and Douglas D. Davis (1990) "The Effects of Non-Binding Price Announcements on Posted Offer Markets,” Economics Letters, 34, 307-310

Isaac, R. Mark, Valerie Ramey and Arlington W. Williams (1984) “Market Organization and Conspiracies in Restraint of Trade” Journal of Economic Behavior and Organization, 5, 191-222.

Kurzban, Robert, and Daniel Houser (2005) "Experiments Investigating Cooperative Types in Humans: A Complement to Evolutionary Theory and Simulations” Proceedings of the National Academy of Science, 102, 1803-1807.

Offerman, T., Sonnemans, J., and Schram, A. (1996) "Value Orientations, Expectations, and Voluntary Contributions in Public Goods.” Economic Journal 106. 817-845.

Potters, Jan and Sigrid Suetens (2008) "Strategic Interaction, Externalities and Cooperation: Experimental Evidence. Working Paper Tilburg University

United States Department of Justice and Federal Trade Commission: Horizontal Merger Guidelines. (1997)

Smith, Vernon L, Gerry L. Suchanek, and Arlington W. Williams (1988) "Bubbles, Crashes and Endogeneous Expectations in Experimental Sport Asset Market," Econometrica, 56, 1119-1152.

Smith, Vernon L. and Arlington W. Williams (1990) "The Boundaries of Competitive Price Theory: Convergence, Expectations and Transactions Costs” in Advances in Behavioral Economics, edited by L. Green and J. Kagel, Ablex Publishing, New York, 1990, 3-35.

Wilcox, N.T. and N. Feltovich (2002): Thinking like a game theorist: comment, Working Paper, University of Houston.

Williams, Arlington W. (1987) "The Formation of Price Forecasts in Experimental Markets” Journal of Money, Credit, and Banking, 19(1), 1-18 


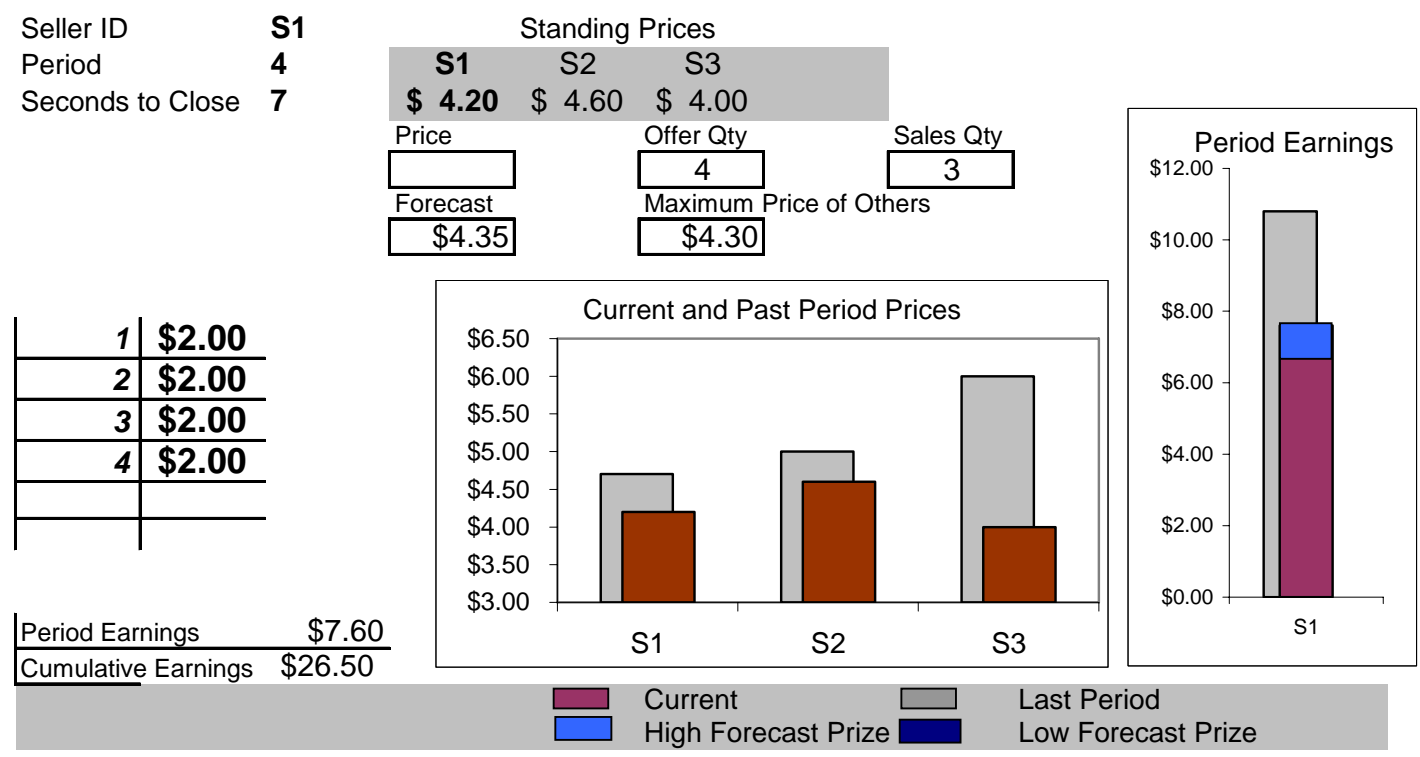

Figure 1. Screen Display for a 'Near Continuous’ Posted Offer Institution

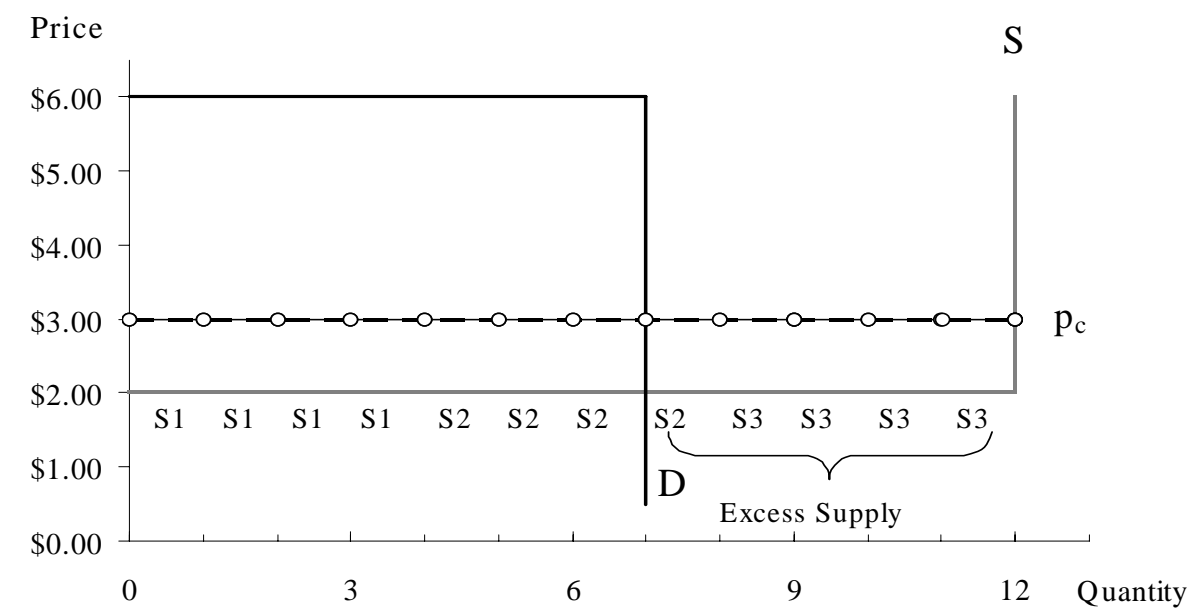

Figure 2: Supply and Demand Arrays for a Three-Seller Swastika Design 

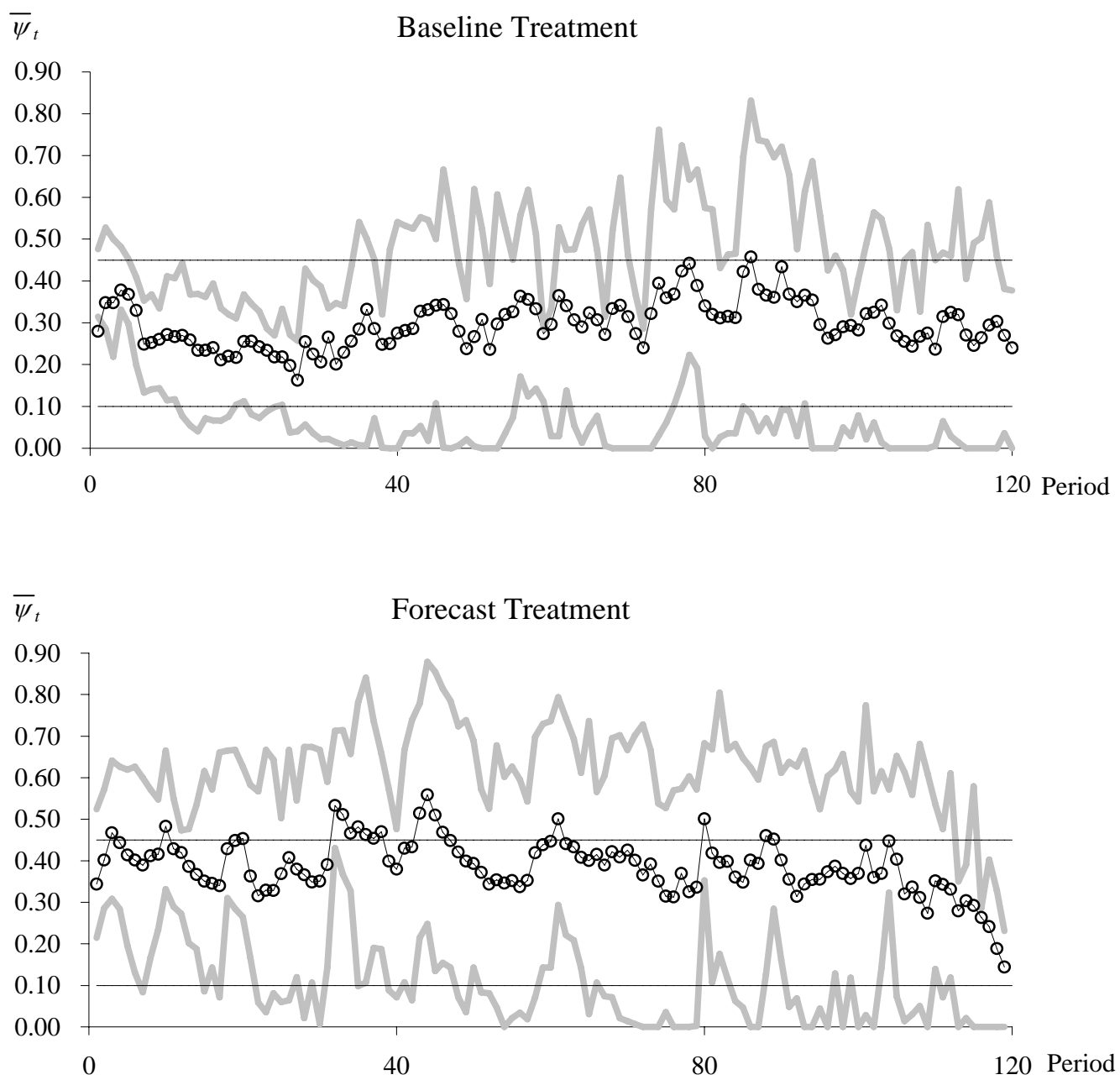

Figure 3. Collusive Efficiencies: Means and Inter-quartile Ranges.

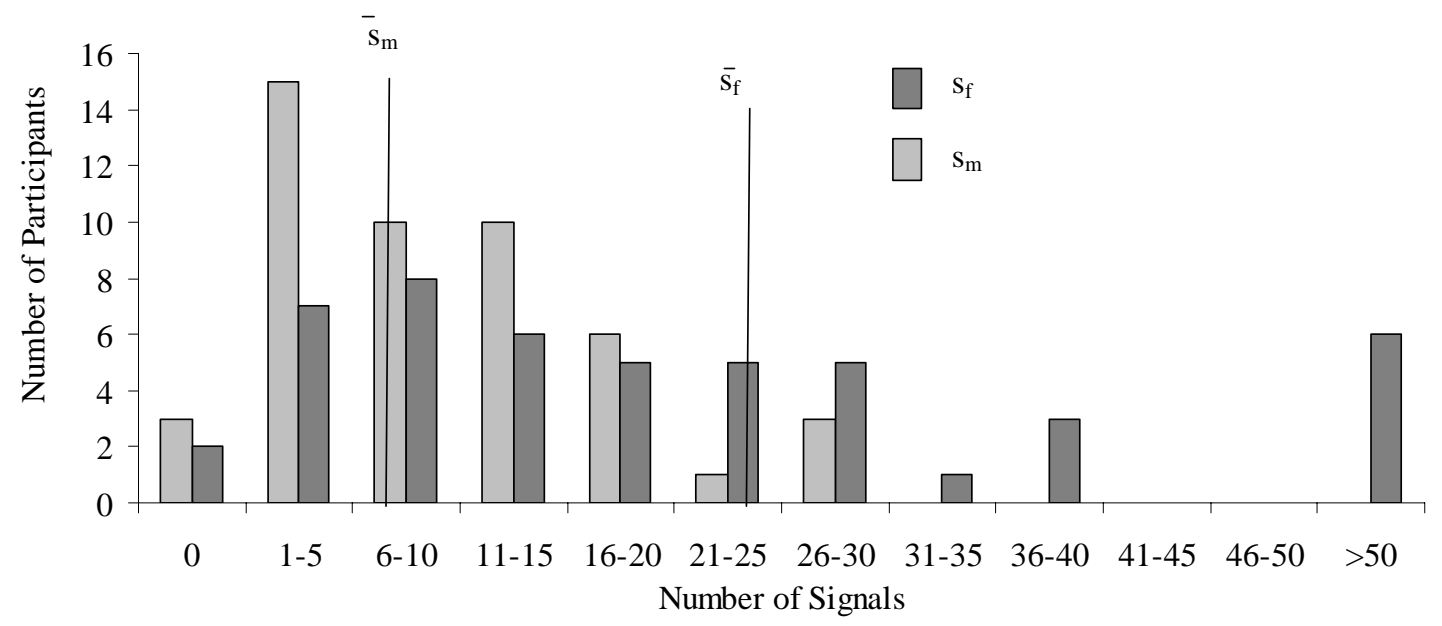

Figure 4. A Comparison of $s_{f}$ and $s_{m}$. 


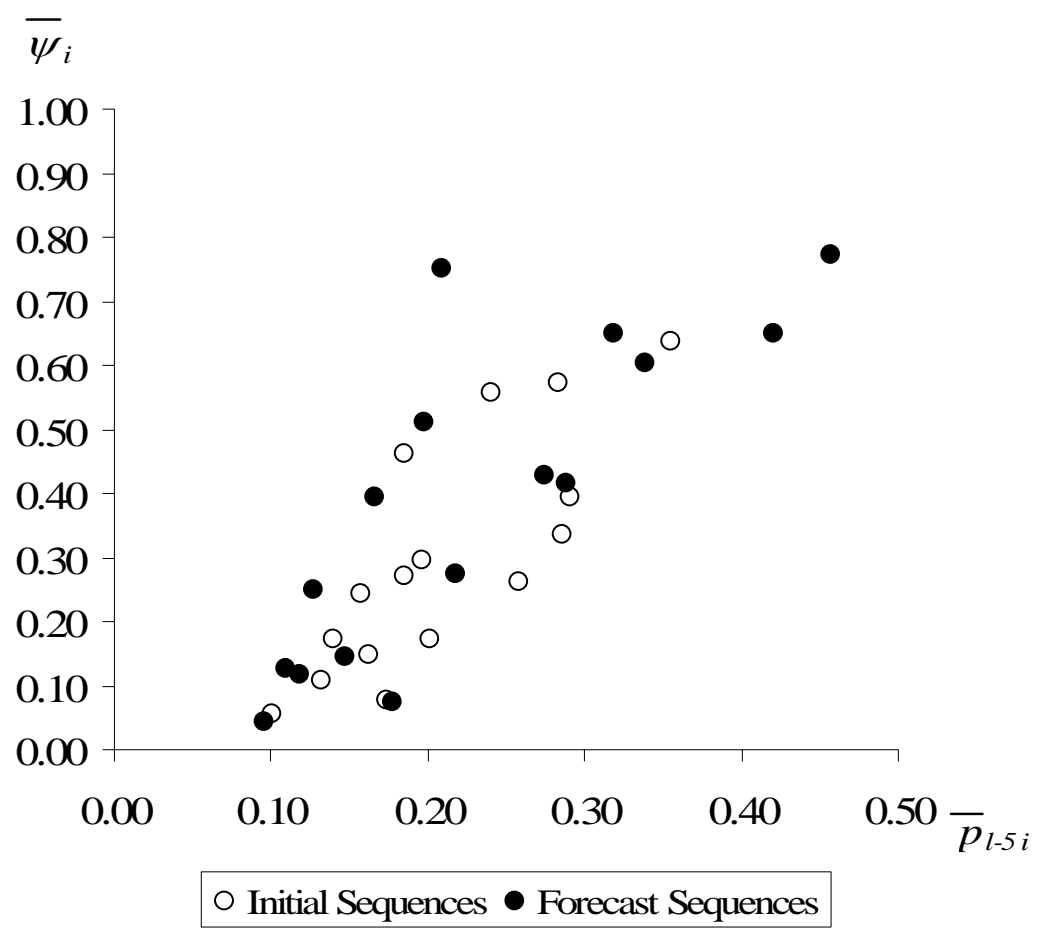

Figure 5. Mean Collusive Efficiencies $\left(\bar{\psi}_{j}\right)$ and Mean Cooperativeness $\left(\bar{p}_{l-5 j}\right)$ of participants in market $j$.

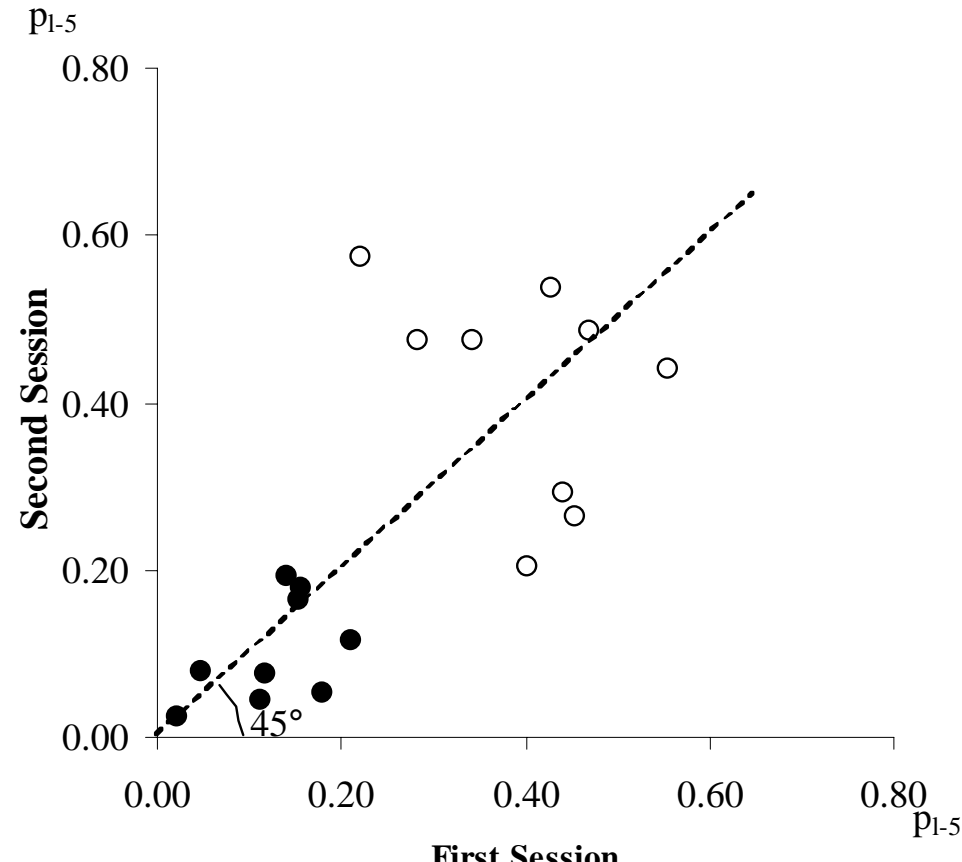

O Cooperative Sellers $\bullet$ Competitive Sellers

Figure 6. $\bar{p}_{l-5, i}$ Cooperativeness Measures across Sessions. 
Table 1. $\bar{\psi}$ (s.d.)

\begin{tabular}{|c|c|c|c|c|c|}
\hline Periods & \multicolumn{2}{|c|}{ Treatment } & \multicolumn{3}{|c|}{$\mathrm{H}_{\mathrm{o}}$} \\
\hline (1) & (2) & (3) & (4) & (5) & (6) \\
\hline & Baseline & Forecast & $\bar{\psi}_{B} \leq .10$ & $\bar{\psi}_{F} \leq .10$ & $\bar{\psi}_{B}=\bar{\psi}_{F}$ \\
\hline $1-60$ & $0.26 \quad(0.15)$ & $(0.24)$ & 0.00 & 0.00 & 0.14 \\
\hline $61-120$ & $0.32 \quad(0.25)$ & $(0.37)$ & 0.00 & 0.00 & 0.60 \\
\hline All & $(0.18)$ & $0.39 \quad(0.39)$ & 0.00 & 0.00 & 0.34 \\
\hline
\end{tabular}

Notes: Entries in columns (4) and (5) are Wilcoxon $p$-values (one tailed tests). Entries in column (6) are Mann Whitney $p$-values (two-tailed tests).

Table 2. Signals and Transactions Prices

Dependent Variable: $\bar{p}_{j t}$

\begin{tabular}{lccc}
\hline & \multicolumn{3}{c}{ Sequence } \\
& Baseline & \multicolumn{2}{c}{ Forecast } \\
& $(1)$ & $(2)$ & $(3)^{* * *}$ \\
Constant & $0.36^{* * *}$ & $0.37^{* * *}$ & $0.51^{* *}$ \\
& $(0.11)$ & $(0.04)$ & $(0.12)$ \\
$\bar{p}_{j t-1}$ & $0.89^{* * *}$ & $0.89^{* * *}$ & $0.86^{* * *}$ \\
& $(0.03)$ & $(0.09)$ & $(0.03)$ \\
$I_{s m}$ & $0.45^{* * *}$ & $0.32^{* * *}$ & \\
& $(0.06)$ & $(0.03)$ & \\
$I_{s f}$ & & & $0.18^{* * *}$ \\
& & & $(0.04)$ \\
Number of clusters & 16 & 16 & 16 \\
Number of observations & 1872 & 1888 & 1872 \\
$\bar{R}^{2}$ & 0.80 & 0.80 & 0.77 \\
$\mathrm{~F}_{(2,15)}$ & $510.48^{* * * *}$ & $1210.45^{* * *}$ & $447.61^{* * *}$ \\
\hline Notes: Robust standard errors in parentheses are adjusted for clustering at the \\
market level. Significant at ${ }^{*}$ 10 percent level; ${ }^{* *} 5$ percent level, ${ }^{* * *} 1$ percent \\
level.
\end{tabular}


Table 3. Comparing $s_{f}$ and $s_{m}$

\begin{tabular}{lll}
\hline Signal Measure & $s_{f}$ & $s_{m}$ \\
\hline (1) Number (average per seller) & $998(21.0)$ & $453(9.4)$ \\
\hline (2) Unintended Signals (\% of Signals) & & $128(28.3 \%)$ \\
\hline (3) Masked Signals (\% of Signals) & $275(27.6 \%)$ & $66(14.6 \%)$ \\
(4) $s_{f}$ signals missed by $s_{m}$ (\% of Signals) & $\begin{array}{l}674(67.4 \%) \\
\text { (5) Correlation }\end{array} \bar{\rho}_{s_{m}, s_{f}}=0.41$ & \\
\hline
\end{tabular}

Key: Masked Signals' under $s_{f}$ are prices less than $\$ 6$ that exceed forecasts, but are below the period's maximum price. Masked signals under $s_{m}$ are prices less than $\$ 6$ that exceed the previous period's maximum price, but are below the maximum posted by others in the current period. 'Unintended' signals under $s_{m}$ are postings below the period forecast, but above the previous period's maximum price. Signals missed by $s_{m}$ are signals sent under $s_{f}$ that were not recorded as signals under $s_{m}$.

Table 4. Mean Collusive Efficiencies by Treatment

\begin{tabular}{cc}
\hline & \\
\cline { 2 - 2 } Competitive $(\mathrm{n}=6)$ & $20.7^{* *}$ \\
Initial Sessions $(\mathrm{n}=32)$ & $34.2^{\dagger \dagger}$ \\
Cooperative $(\mathrm{n}=6)$ & $76.4^{* *}+\dagger$ \\
${ }^{++,{ }^{* *}}$ Unequal (within column comparisons) using a two-tailed Mann Whitney test $\mathrm{p}<.01$
\end{tabular}

